\title{
A Fast Simplified Piecewise Constant Doppler Algorithm for Generalized Continuous Wave Synthetic Aperture Radar
}

\author{
Yijiang Nan \\ University of Technology Sydney \\ Sydney, Australia \\ yijiang.nan@student.uts.edu.au
}

\author{
Xiaojing Huang \\ University of Technology Sydney \\ Sydney, Australia \\ Xiaojing.Huang@uts.edu.au
}

\author{
Y. Jay Guo \\ University of Technology Sydney \\ Sydney, Australia \\ jay.guo@uts.edu.au
}

\begin{abstract}
With the removal of the slow time, generalized continuous wave synthetic aperture radar (GCW-SAR) adopts the one-dimensional data imaging, offering many advantages compared with the conventional SAR system. In this paper, a fast simplified piecewise constant Doppler algorithm is proposed based on the further zero-th order approximation on top of the linear approximation of the slant range, leading to a flexible azimuth imaging spacing. Hence, the reduction of the complexity can be obtained by extending the azimuth imaging spacing and downsampling the received signal in digital domain. Simulation results can validate the advantages of the proposed algorithm.

Index Terms-Synthetic aperture radar, piecewise constant Doppler algorithm, zero-th order approximation.
\end{abstract}

\section{INTRODUCTION}

Synthetic aperture as a high resolution remote sensing technique capable of working day and night under all the weather conditions has been widely used in many civil and defence applications [1]-[4]. In conventional SAR systems, two-dimensional data acquisition within the fast and slow time is adopted, by which the imaging in range and azimuth can be achieved by the time delay of the backscattered signal and the different Doppler frequency shift between the radar and targets respectively[].

Recently, a generalized continuous wave synthetic aperture radar (GCW-SAR) [5] is proposed for the one-dimensional data imaging with the removal of the slow time, and thus some intrinsic limitations caused by the slow time can be eliminated. In GCW-SAR, the radar transmits a continuous wave signal over the entire synthetic aperture time and receives its backscattered signals simultaneously after the selfinterference cancellation. The GCW-SAR imaging process in principle adopts the time correlation method or matched filtering approach known as the back-projection algorithm (BPA). For each location of the image, its location dependent reference signal is firstly produced and then the corresponding image pixel is achieved by performing the correlation of the received signal with its location dependent reference signal.

Unlike two-dimensional data structure in conventional SAR, the range and azimuth information is mixed and cannot be

This work is supported by the Australia Research Council Discovery Project 160101693 separated. Hence, the existing SAR algorithms, e.g., frequency domain algorithms [4], fast BPAs [6], [7], based on the slow time sampling cannot be used in GCW-SAR. To reduce the complexity of the one-dimensional data imaging process, the piecewise constant Doppler (PCD) algorithm is proposed based on the piecewise linear approximation of the slant range. The Doppler frequency shift between two adjacent targets in azimuth over each linear segment becomes a constant and thus the time correlation over each segment can be derived recursively from the outcome of the previous one, building up a fast recursive radar imaging algorithm. In PCD algorithm, the azimuth imaging spacing is equivalent to the radar travelling distance over a sampled period. Since the sampling rate is much larger than the Doppler bandwidth, there are many redundant pixels to be imaged in azimuth direction. Such useless imaging process leads to a high computational complexity and hard applications in practice.

In this paper, a fast simplified PCD algorithm is proposed based on the further zero-th order approximation of the linear segment in the PCD algorithm. Since the slant range becomes constant during each constant segment, the correlation of the received samples with the reference samples can be accumulated before the recursive process, thus forming a flexible azimuth imaging spacing in the PCD algorithm. On the premise of the imaging quality, the extending imaging spacing can reduce the number of redundant PCD imaging pixels and lead to a significant reduction of computational cost. On the other hand, the computational cost can be further reduced by downsampling the received signal in digital domain since the reduction of the received samples used in the correlation over the zero-th order segment is independent of the recursive imaging due to the constant slant range. Note that the downsampling the received signal may lead to a lower signal-to-noise ratio (SNR) in the final PCD image.

This paper is organized as follows: Section II revisits the PCD principle, develops the proposed fast simplified PCD algorithm and analyzes the complexity. Section III shows the simulation results and the conclusion is drawn in Section V. 


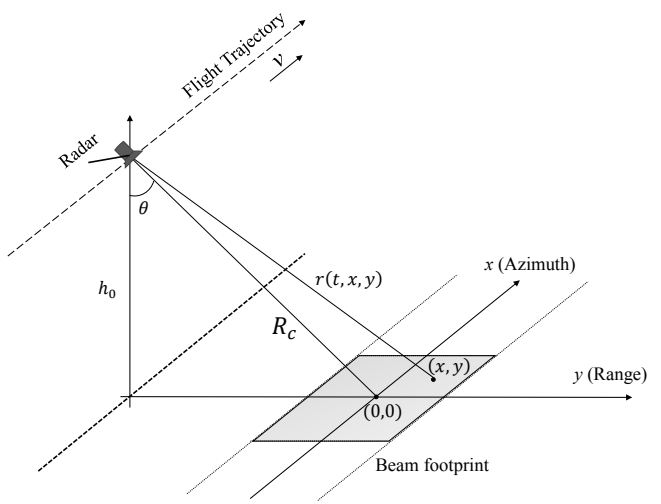

Fig. 1: Stirpmap SAR geometry.

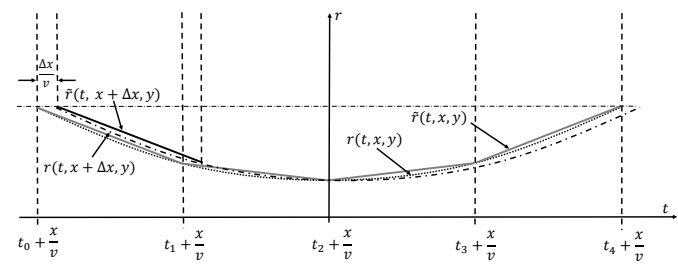

Fig. 2: Linear approximation in the PCD algorithm.

\section{FAST Simplified PCD ALGORITHM}

Since any forms of the slant range can be approximated as linear segments, the PCD algorithm can be used in any radar imaging system with the knowledge of the radar trajectory. To describe the proposed PCD algorithm more clearly, a common stripmap SAR geometry is adopted in this paper, as shown in Fig. 1. The side-looking radar at a height $h$ travels along the $x$-axis in a straight line with a constant speed $v$. The beam footprint center is set to the origin $(0,0)$. The $R_{c}$ and $r(t, x, y)$ are the distances from the radar to the origin and point $(x, y)$ respectively. Note that only a flat terrain is considered for simplicity.

In this mode, the demodulated received signal can be described as

$$
s_{r}(t)=\iint \sigma(x, y) s_{t}\left(t-\frac{2 r(t, x, y)}{c}\right) e^{-j \frac{4 \pi}{\lambda} r(t, x, y)} d x d y
$$

where the $\sigma(x, y)$ is the radar cross section (RCS) of the target located at $(x, y), \lambda$ is the transmitted wavelength and $s_{t}(t)$ is the transmitted signal. The instantaneous slang range $r(t, x, y)$ can be expressed as

$$
r(t, x, y)=\sqrt{\left(R_{c} \sin \theta+y\right)^{2}+(x-v t)^{2}+h_{0}^{2}} .
$$

\section{A. PCD principle}

Theoretically speaking, the PCD algorithm is designed as a fast $\mathrm{BP}$ algorithm for the one-dimensional data imaging. The slant range $r(t, x, y)$ is assumed to be divided into $P$ linear segments as shown in Fig. 2, where the $P$ is set to 4 for simplicity. The time correlation imaging result $I(x, y)$ for the point $(x, y)$ can be expressed as a summation of that over different linear segments, i.e., $I(x, y)=\sum_{p=0}^{P-1} I^{(p)}(x, y)$. Assuming that time duration over each linear segment is set to $T_{P}$ and the $p$-th segment of any point at the range line $(0, y)$ starts from the time $t_{p}$, where $p=0,1,2, \ldots, P-1$, the approximated slant range $\widetilde{r}(t, x, y)$ can be expressed as

$$
\begin{aligned}
& \widetilde{r}(t, x, y) \\
& =\frac{r\left(t_{p+1}, 0, y\right)-r\left(t_{p}, 0, y\right)}{\left(t_{p+1}-t_{p}\right)}\left(t-t_{p}-\frac{x}{v}\right)+r\left(t_{p}, 0, y\right) \\
& =-\frac{\lambda}{2} f_{D_{p}}(y)\left(t-t_{p}-\frac{x}{v}\right)+r\left(t_{p}, 0, y\right) \\
& , t \in\left[t_{p}+\frac{x}{v}, t_{p+1}+\frac{x}{v}\right)
\end{aligned}
$$

where $r\left(t+\frac{x}{v}, x, y\right)=r(t, 0, y)$ is valid in the flat terrain and $f_{D_{p}}\left(y_{n}\right)=-\frac{2}{\lambda} \frac{r\left(t_{p+1}, 0, y_{n}\right)-r\left(t_{p}, 0, y_{n}\right)}{t_{p+1}-t_{p}}$ is the constant Doppler frequency for the $p$-th segment.

As shown in Fig. 2, the difference between the approximated instantaneous slant ranges $\widetilde{r}(t, x, y)$ and $\widetilde{r}(t, x+\Delta x, y)$ of two adjacent azimuth points $(x, y)$ and $(x+\Delta x, y)$, plotted by the grey and black solid line respectively, remains constant over each linear segment. If the received signal satisfies the condition $s_{t}\left(t+\frac{x}{v}-\frac{2 r(t, \Delta x, y)}{c}\right) \approx s_{t}\left(t+\frac{x}{v}-\frac{2 r(t, 0, y)}{c}\right)$, the $I^{(p)}(x+\Delta x, y)$ can be derived recursively from the $I^{(p)}(x, y)$, expressed as

$$
\begin{aligned}
& I^{(p)}(x+\Delta x, y) \\
& =\int_{\frac{\Delta x}{v}+t_{p}}^{\frac{\Delta x}{v}+t_{p+1}} s_{r}\left(t+\frac{x}{v}\right) s_{t}^{*}\left(t+\frac{x}{v}-\frac{2 \widetilde{r}(t, \Delta x, y)}{c}\right) \\
& \cdot e^{j \frac{4 \pi}{\lambda} \widetilde{r}\left(t+\frac{x}{v}, x+\Delta x, y_{n}\right)} d t \\
& \approx \int_{t_{p}}^{t_{p+1}} s_{r}\left(t+\frac{x}{v}\right) s_{t}^{*}\left(t+\frac{x}{v}-\frac{2 \widetilde{r}(t, 0, y)}{c}\right) \\
& \cdot e^{j \frac{4 \pi}{\lambda}\left(-\frac{\lambda}{2} f_{D_{p}}(y)\left(t-t_{p}\right)+r\left(t_{p}, 0, y\right)\right)} e^{j 2 \pi f_{D_{p}}(y) \frac{\Delta x}{v}} d t \\
& -\int_{t_{p}}^{\frac{\Delta x}{v}+t_{p}} s_{r}\left(t+\frac{x}{v}\right) s_{t}^{*}\left(t+\frac{x}{v}-\frac{2 \widetilde{r}(t, \Delta x, y)}{c}\right) \\
& \cdot e^{j \frac{4 \pi}{\lambda} \widetilde{r}(t, \Delta x, y)} d t \\
& +\int_{t_{p+1}}^{\frac{\Delta x}{v}+t_{p+1}} s_{r}\left(t+\frac{x}{v}\right) s_{t}^{*}\left(t+\frac{x}{v}-\frac{2 \widetilde{r}(t, \Delta x, y)}{c}\right) \\
& \cdot e^{j \frac{4 \pi}{\lambda} \widetilde{r}(t, \Delta x, y)} d t \\
& =I^{(p)}(x, y) e^{j 2 \pi f_{D_{p}}}(y) \frac{\Delta x}{v} \\
& -\int_{t_{p}}^{\frac{\Delta x}{v}+t_{p}} s_{r}\left(t+\frac{x}{v}\right) s_{t}^{*}\left(t+\frac{x}{v}-\frac{2 \widetilde{r}(t, \Delta x, y)}{c}\right) \\
& \cdot e^{j \frac{4 \pi}{\lambda} \widetilde{r}(t, \Delta x, y)} d t \\
& +\int_{t_{p+1}}^{\frac{\Delta x}{v}+t_{p+1}} s_{r}\left(t+\frac{x}{v}\right) s_{t}^{*}\left(t+\frac{x}{v}-\frac{2 \widetilde{r}(t, \Delta x, y)}{c}\right) \\
& \cdot e^{j \frac{4 \pi}{\lambda} \widetilde{r}(t, \Delta x, y)} d t
\end{aligned}
$$

where the $*$ represents the conjugate operation. Obviously, the recursive process in the PCD algorithm is composed of three steps: compensating the Doppler frequency shift, removing the useless integral and adding the new integral. 


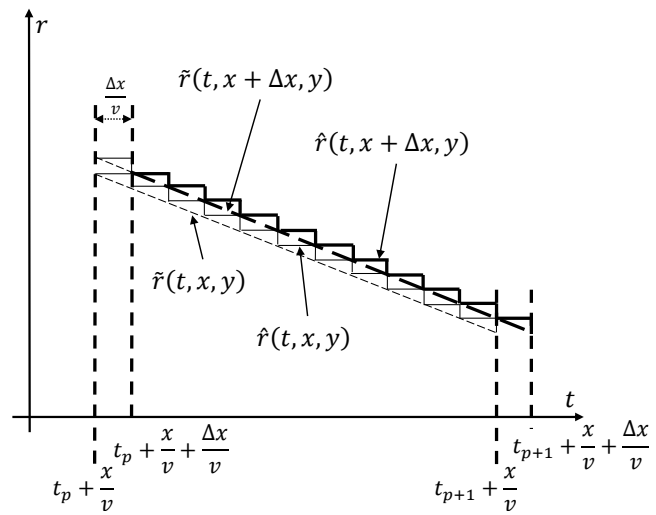

Fig. 3: Zero-th order approximation of the linear segment in the PCD algorithm.

\section{B. Simplified PCD Algorithm}

In the original PCD algorithm, the recursive process is performed whenever the GCW-SAR system received a sample, thus producing many redundant pixels in the final SAR image [5]. In this paper, the zero-th order approximation of the linear segment in the PCD algorithm is adopted as seen in Fig. 3, where the further approximated slant range is described as $\widehat{r}(t, x, y)$ and the $I^{(p)}(x+\Delta x, y)$ can be simplified as

$$
\begin{aligned}
& I^{(p)}(x+\Delta x, y) \\
& =I^{(p)}(x, y) e^{j 2 \pi f_{D_{p}}(y) \frac{\Delta x}{v}}-e^{j \frac{4 \pi}{\lambda} \widehat{r}\left(t_{p}, \Delta x, y\right)} \\
& \cdot \int_{t_{p}}^{\frac{\Delta x}{v}+t_{p}} s_{r}\left(t+\frac{x}{v}\right) s_{t}^{*}\left(t+\frac{x}{v}-\frac{2 \widehat{r}(t, \Delta x, y)}{c}\right) d t \\
& +e^{j \frac{4 \pi}{\lambda} \widehat{r}\left(t_{p}, \Delta x, y\right)} \int_{t_{p+1}}^{\frac{\Delta x}{v}+t_{p+1}} s_{r}\left(t+\frac{x}{v}\right) \\
& \cdot s_{t}^{*}\left(t+\frac{x}{v}-\frac{2 \widehat{r}(t, \Delta x, y)}{c}\right) d t \\
& =I^{(p)}(x, y) e^{j 2 \pi f_{D_{p}}(y) \frac{\Delta x}{v}}-e^{j \frac{4 \pi}{\lambda} r\left(t_{p}, \Delta x, y\right)} \\
& \cdot \int_{t_{p}}^{\frac{\Delta x}{v}+t_{p}} s_{r}\left(t+\frac{x}{v}\right) s_{t}^{*}\left(t+\frac{x}{v}-\frac{2 r\left(t_{p}, \Delta x, y\right)}{c}\right) d t \\
& +e^{j \frac{4 \pi}{\lambda} r\left(t_{p}, \Delta x, y\right)} \int_{t_{p+1}}^{\frac{\Delta x}{v}+t_{p+1}} s_{r}\left(t+\frac{x}{v}\right) \\
& \cdot s_{t}^{*}\left(t+\frac{x}{v}-\frac{2 r\left(t_{p+1}, \Delta x, y\right)}{c}\right) d t
\end{aligned}
$$

where the $\widehat{r}(t, x, y)=r\left(t_{p}, x, y\right)$ when $t \in\left[t_{p}+\frac{x}{v}, t_{p}+\frac{x}{v}+\frac{\Delta x}{v}\right)$ and the correlation of the received signal with the reference signal can be accumulated before the recursive process, leading to an extended azimuth imaging spacing.

Assuming that the linear segment is further divided into $K$ constant segments and the sampling period is set to $T_{s}$, the azimuth imaging spacing $\Delta x$ can be extended from $T_{s} v$ to $T_{P} / K * v=N T_{s} v$, where $N$ represents the number of the received samples over each constant segment. The discrete azimuth imaging location $x$ can be set to $m \Delta x$ and the recursive process can be expressed as

$$
\begin{aligned}
& I^{(p)}((m+1) \Delta x, y) \\
& =I^{(p)}\left(m \Delta x, y_{n}\right) e^{j 2 \pi f_{D_{p}}\left(y_{n}\right) N T_{s}}-e^{j \frac{4 \pi}{\lambda} r\left(t_{p}, \Delta x, y\right)} \\
& \cdot T_{s} \sum_{i=1}^{N} s_{r}\left((i+m N) T_{s}+t_{p}\right) s_{t}^{*}\left((i+m N) T_{s}+t_{p}\right. \\
& \left.-\frac{2 r\left(t_{p}, \Delta x, y\right)}{c}\right)+e^{j \frac{4 \pi}{\lambda} r\left(t_{p+1}, \Delta x, y\right)} T_{s} \sum_{i=1}^{N} s_{r}\left((i+m N) T_{s}\right. \\
& \left.+t_{p+1}\right) s_{t}^{*}\left((i+m N) T_{s}+t_{p+1}-\frac{2 r\left(t_{p+1}, \Delta x, y\right)}{c}\right) .
\end{aligned}
$$

To further reduce the computational cost, the two summation in (6) can be simplified by downsampling the received signal. Assuming that the number of the received samples in each constant segment is downsampled to $N_{d}=N / D_{r}$, where $D_{r}$ denotes the downsampling rate, Eq. (6) can be derived as

$$
\begin{aligned}
& I^{(p)}((m+1) \Delta x, y) \\
& =I^{(p)}\left(m \Delta x, y_{n}\right) e^{j 2 \pi f_{D_{p}}\left(y_{n}\right) N T_{s}}-e^{j \frac{4 \pi}{\lambda} r\left(t_{p}, \Delta x, y\right)} \\
& \cdot T_{s} D_{r} \sum_{i=1}^{N_{d}} s_{r}\left(\left(i D_{r}+m N\right) T_{s}+t_{p}\right) s_{t}^{*}\left(\left(i D_{r}+m N\right) T_{s}+t_{p}\right. \\
& \left.-\frac{2 r\left(t_{p}, \Delta x, y\right)}{c}\right)+e^{j \frac{4 \pi}{\lambda} r\left(t_{p+1}, \Delta x, y\right)} T_{s} D_{r} \sum_{i=1}^{N} s_{r}\left(\left(i D_{r}+m N\right)\right. \\
& \left.\cdot T_{s}+t_{p+1}\right) s_{t}^{*}\left(\left(i D_{r}+m N\right) T_{s}+t_{p+1}-\frac{2 r\left(t_{p+1}, \Delta x, y\right)}{c}\right) .
\end{aligned}
$$

where the computational cost for the two summations are reduced by $D_{r}$ times. Note that there is trade-off between the downsampling rate and the SNR in the final image. A proper selection of the $D_{r}$ is of great significance in the proposed algorithm.

\section{Complexity}

We compare the number of complex multiplications between the original PCD algorithm and the simplified PCD algorithm when reconstructing the image of the same observed area, where the number of samples within a sweep of the observed area is $N_{s}^{2}$ and the number of range pixels is $N_{s}$. The recursive process in the PCD algorithm operates whenever the radar receives a new demodulated sample, thus achieving an $N_{s} \times N_{s}^{2}$ pixel image over the observed scene. Hence, the total number of the complex multiplications in the original PCD algorithm is $(3 P+2) \times N_{s}^{3}$, where each pixel costs $3 P+2$ complex multiplications [5]. By comparison, the proposed PCD algorithm only achieves an $N_{s} \times N_{s}^{2} / D_{r}$ pixel image and the received signal is downsampled, leading to a lower complexity. Therefore, the number of complex multiplications in the proposed PCD algorithm is reduced to $(P+1) \cdot N_{s}^{2} \cdot N_{s} / D_{r}+(2 P+1) N^{3} / N_{s}$, where the time correlation costs $(P+1) \cdot N_{s}^{2} \cdot N_{s} / D_{r}$ complex multiplications and the recursive process costs $(2 P+1) N^{3} / N_{s}$. 


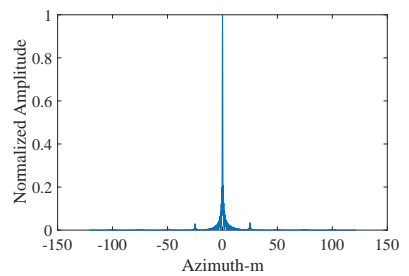

(a)

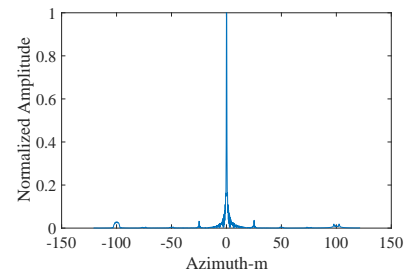

(c)

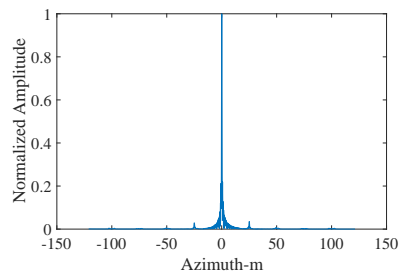

(b)

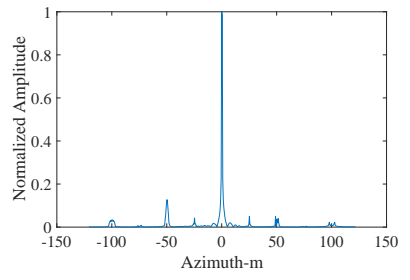

(d)
Fig. 4: Proposed PCD imaging results in azimuth with different $K:$ (a) $K=40$, (b) $K=30$, (c) $K=20$ and (d) $K=10$.

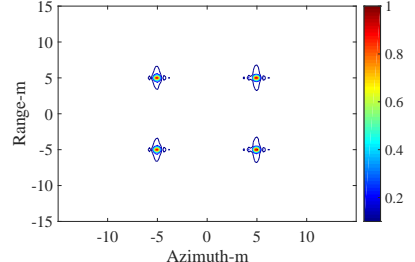

(a)

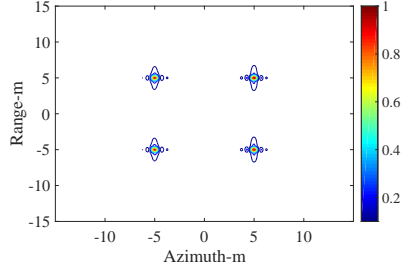

(b)
Fig. 5: Multi-targets PCD images with different $K$ : (a) $K=30$ and (b) $K=200$.

\section{Simulation Results}

In this section, the PCD algorithm is evaluated based on the stripmap airborne SAR system, where the radar height is $h_{0}=7000 \mathrm{~m}$, the carry frequency is $f_{c}=10 \mathrm{GHz}$, the antenna aperture is $1 \mathrm{~m}$, speed of light is $c=3 \times 10^{8} \mathrm{~m} / \mathrm{s}$, the radar platform speed is $70 \mathrm{~m} / \mathrm{s}$, and the beam incident angle is $30 \mathrm{de}-$ gree. Assuming that the radar transmits continuous wave chirp signal periodically with frequency bandwidth $B=100 \mathrm{MHz}$ and the number of the linear segments in the PCD algorithm is set to $P=50$. In the PCD algorithm, the image pixels in range are reconstructed independently without any approximation, and thus the approximation of the slant range only degrade the image quality in azimuth.

The first experiment investigates the PCD imaging quality when considering different number of constant segments $K$. The image with one-point target at the origin is adopted and the azimuth imaging result within $K=40,30,20,10$ are shown in Fig. 4 (a), (b), (c), and (d) respectively. It is evident that the imaging ambiguities caused by the zero-th order approximation of the linear segments is reduced with the increase of $K$ since the shorter length of the constant

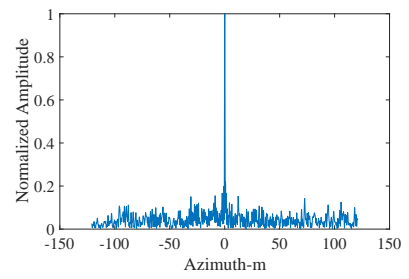

(a)

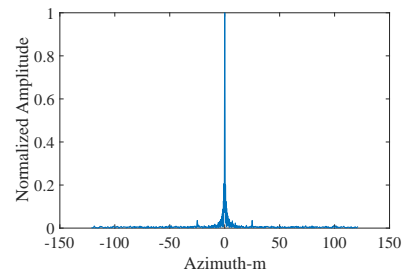

(c)

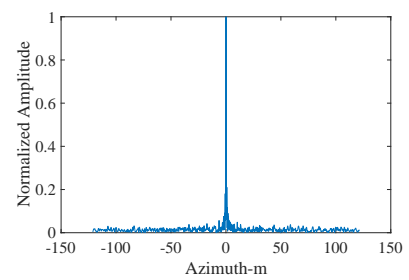

(b)

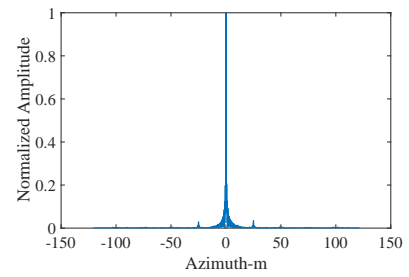

(d)
Fig. 6: Proposed PCD imaging results in azimuth with different $D_{r}$ : (a) $D_{r}=10000$, (b) $D_{r}=1000$, (c) $D_{r}=100$ and (d) $D_{r}=10$.

segment leads to a more accurate approximation. However, when $K$ is large enough, the image quality can be hardly improved. Comparing the image quality between the cases of $K=40$ and $K=30$, nearly the same images are obtained, which can be also validated by the multi-targets PCD imaging case, as seen in Fig. 5 where the PCD images with $K=30$ and $K=200$ are shown respectively. The trade-off between the downsampling rate $D_{r}$ and the SNR is investigated in the second experiment. Assuming that the number of constant segments $K$ is set to 40 and the SNR is set to $-30 \mathrm{~dB}$, the azimuth imaging results with different $D_{r}=10000,1000,100,10$ are presented respectively in Fig. 6. With the decrease of $D_{r}$, the PCD image with a lower noise energy can be achieved. Therefore, the number of the constant segments $K$ and downsampling rate $D_{r}$ should be selected properly to balance the image quality and the computational cost.

\section{CONCLUSION}

This paper develop a fast simplified PCD algorithm for GCW-SAR system. The PCD principle where the slant range is approximated as linear segments is firstly revealed. To reduce the number of redundant image pixels and computational cost, the zero-th order approximation of the linear segments in the PCD algorithm is then developed and the reduced complexity is analyzed accordingly. Finally, the simulation results are presented which can validate the proposed algorithm. The PCD algorithms can be widely used in any radar imaging system with the known trajectory of the radar. The proposed algorithm extends the azimuth imaging spacing and reduces the complexity, thus simplifying the PCD algorithm. 


\section{ACKNOWLEDGMENT}

This work was supported under the School of Electrical and Data Engineering in University of Technology, Sydney and the Australian Research Council (ARC) Discovery Project.

\section{REFERENCES}

[1] X. M. Li, S. Lehner, and T. Bruns, "Ocean Wave Integral Parameter Measurements Using Envisat ASAR Wave Mode Data," IEEE Transactions on Geoscience and Remote Sensing, vol. 49, no. 1, pp. 155-174, Jan 2011.

[2] J. H. Gonzalez, M. Bachmann, R. Scheiber, and G. Krieger, "Definition of ICESat Selection Criteria for Their Use as Height References for TanDEM-X," IEEE Transactions on Geoscience and Remote Sensing, vol. 48, no. 6, pp. 2750-2757, June 2010.

[3] L. B. Wang, D. W. Wang, J. J. Li, J. Xu, C. Xie, and L. Wang, "Ground Moving Target Detection and Imaging Using a Virtual Multichannel Scheme in HRWS Mode," IEEE Transactions on Geoscience and Remote Sensing, vol. 54, no. 9, pp. 5028-5043, Sept 2016.

[4] I. G. Cumming and F. H. Wong, Digital processing of synthetic aperture radar data, 2005, vol. 1, no. 2.

[5] Y. Nan, X. Huang, and Y. J. Guo, "Generalized continuous wave synthetic aperture radar for high resolution and wide swath remote sensing," IEEE Transactions on Geoscience and Remote Sensing, vol. 56, no. 12, pp. 7217-7229, Dec 2018.

[6] L. Zhang, H. Li, Z. Qiao, and Z. Xu, "A fast bp algorithm with wavenumber spectrum fusion for high-resolution spotlight sar imaging," IEEE Geoscience and Remote Sensing Letters, vol. 11, no. 9, pp. 14601464, Sep. 2014.

[7] Y. Luo, F. Zhao, N. Li, and H. Zhang, "An autofocus cartesian factorized backprojection algorithm for spotlight synthetic aperture radar imaging," IEEE Geoscience and Remote Sensing Letters, vol. 15, no. 8, pp. 12441248, Aug 2018. 